\title{
Pistacia lentiscus L. edible oil: green extraction with bio-based solvents, metabolite profiling and in vitro anti-inflammatory activity $^{\text {光 }}$
}

\author{
Emna Chaabani ${ }^{1,2,3}$, Maryline Abert Vian ${ }^{1, *}$, Sarra Dakhlaoui ${ }^{2}$, Soumaya Bourgou ${ }^{2}$, Farid Chemat ${ }^{1}$ and \\ Riadh Ksouri ${ }^{2}$ \\ ${ }^{1}$ Université d'Avignon et des Pays de Vaucluse, INRA, UMR408, GREEN Extraction Team, 84000 Avignon, France \\ ${ }^{2}$ Laboratoire des Plantes Aromatiques et Médicinales (LPAM), Centre de Biotechnologie de Borj-Cédria, BP 901, 2050 Hammam-lif, \\ Tunisia \\ ${ }^{3}$ Faculté des Sciences de Bizerte, Département Sciences Biologiques, Jarzouna 7020, Bizerte, Tunisia
}

Received 7 March 2019 - Accepted 2 May 2019

\begin{abstract}
This study assesses, qualitatively and quantitatively, the performance of ten bio-based solvents to replace hexane for the extraction of oil from Pistacia lentiscus edible fruits. Solvent screening was achieved through theoretical approach using the COnductor-like Screening MOdel for Realistic Solvation (COSMO-RS) predictions and an experimental protocol based on GC-FID and HPTLC analysis. Unsupervised multivariate analyses were performed on GC-FID and HPTLC data. Experiments corroborated theoretical predictions indicating that MeTHF was the best alternative solvent to replace hexane. Moreover, the obtained extract exhibited a good anti-inflammatory activity, inhibiting by $91.9 \% \mathrm{NO}$ release in RAW 264.7 macrophages. These findings highlighted the potential of green solvents especially MeTHF in lipid extraction and exhibited that $P$. lentiscus oil presents a potential anti-inflammatory property and great nutritional value, notably high amount of oleic and linoleic acid, thus encouraging its valorization in food, cosmetic or pharmaceutical industry.
\end{abstract}

Keywords: Pistacia lentiscus L / oil extraction / bio-based solvents / COSMO-RS / anti-inflammatory activity

\begin{abstract}
Résumé - L'huile comestible de Pistacia lentiscus L.: éco-extraction par des agro-solvants, profilage métabolique et activité anti-inflammatoire in vitro. Cette étude consiste à évaluer, qualitativement et quantitativement, la performance de dix agro-solvants afin de remplacer l'hexane pour l'extraction de l'huile à partir des fruits comestibles de Pistacia lentiscus. Le criblage des solvants a été réalisé par une approche théorique en utilisant des outils de prédiction tels que COSMO-RS et une approche expérimentale basée sur les analyses CG-DIF et HPTLC. Des analyses multivariées ont été effectuées sur les données CG-DIF et HPTLC. Les résultats expérimentaux ont confirmé les prédictions théoriques indiquant que le MeTHF était le meilleur solvant alternatif pour substituer l'hexane. En outre, l'extrait obtenu présentait une bonne activité anti-inflammatoire, inhibant de $91,9 \%$ la libération de NO dans les macrophages RAW 264.7. Ces résultats ont mis en évidence le potentiel des solvants verts, en particulier du MeTHF dans l'extraction lipidique et ont montré que l'huile de $P$. lentiscus présentait une activité antiinflammatoire potentielle et une grande valeur nutritionnelle, notamment une teneur élevée en acide oléique et linoléique, encourageant ainsi sa valorisation dans les industries alimentaire, cosmétique ou pharmaceutique.
\end{abstract}

Mots clés : Pistacia lentiscus L / extraction d'huile / agro-solvants / COSMO-RS / activité anti-inflammatoire

\footnotetext{
Contribution to the Topical Issue "Technological challenges in oilseed crushing and refining / Défis technologiques de la trituration et du raffinage des oléagineux"

*Correspondence: maryline.vian@univ-avignon.fr
} 


\section{Introduction}

Pistacia lentiscus L. (Lentisk) belongs to the Anacardiaceae family. Lentisk fruits yield an edible oil with a high nutritional value due to its richness in monounsaturated fatty acids, omega 3 fatty acid, $\beta$-sitosterol, $\alpha$-tocopherols and minerals (Mezni et al., 2014). This edible oil has been used traditionally by Tunisians in their daily diet as condiment, in salads and pastries (Trabelsi et al., 2012). The Pistacia lentiscus fruit is used as a source of medicinal oil for the treatment of many diseases such as scabies, respiratory allergies and rheumatism and also for the fabrication of antidiarrhea pills (Ben Khedir et al., 2017). Recently, several studies have reported the pharmacological virtues of this fixed oil (Mezni et al., 2016; Naouar et al., 2016; Ben Khedir et al., 2017).

Conventionally, oils and lipids are extracted using hexane as solvent. Nevertheless, this flammable solvent, derived from petroleum, has recently been categorized as reprotoxic category 2 substance under the REACH regulation (European Directives and Registration, Evaluation, Authorization and Restriction of Chemicals), as category 2 aquatic chronic toxic and listed as forbidden substances in cosmetic products (Rapinel et al., 2016). Nowadays, the development of green chemistry has prompted chemists to find alternative solvents to hazardous ones (Kerton and Marriott, 2013).

Bio-based solvents are renewable resource produced from natural source such as wood, starch, fruits and vegetable oils. These green solvents have a good solvation power. Also, they are biodegradable, non-toxic and non-flammable. Indeed, many studies have shown the potential of alternative solvents for lipid extraction instead of hexane (Sicaire et al., 2015; Breil et al., 2016; Cascant et al., 2017).

In this study, the effectiveness of ten bio-based solvents to substitute hexane for the extraction of lipids from Lentisk berries was evaluated through a computational approach, using COSMO-RS software, and an experimental approach based on GC-FID and HPTLC analysis. Then, anti-inflammatory activity of the best alternative solvent was assessed in vitro on murine macrophages.

\section{Materials and methods}

\subsection{Reagents and solvents}

Among hydrophobic solvents, ten green alternatives were selected from different chemical families. These are represented by two alcohols (ethanol $[\mathrm{EtOH}]$ and isopropanol [IPA]), three esters (ethyl acetate [EtOAc)], ethyl lactate and dimethyl carbonate [DMC]), three terpenes ( $p$-cymene, $\alpha$-pinene and limonene) and two ethers (methyltetrahydrofuran $[\mathrm{MeTHF}]$ and cyclopentyl methyl ether [CPME]). Hexane, taken as reference in this study, EtOH, IPA, MeTHF (purity 99.5\%), DMC (purity 99\%), EtOAc (purity 99.5\%) and limonene were purchased from VWR International (Darmstadt, Germany). $p$-Cymene was sourced from Acros (Hampton, NH, USA). CPME (reagent plus 99.5\%) was obtained from Sigma Aldrich (Sigma Co., St. Louis, MO, USA). Ethyl lactate and $\alpha$-pinene were supplied from Merck (Darmstadt, Germany). All solvents used in extraction were of technical grade.
Hexane of HPLC grade, methanol, chloroform, propan-2-ol, diethyl ether, methyl acetate, acetic acid, sulfuric acid, potassium chloride, sodium chloride and water were of analytical grade and were purchased from VWR International (Darmstadt, Germany). Primuline and acetone were of analytical grade and were provided by Sigma Aldrich. Fatty acid, neutral and polar lipid standards were acquired from Sigma Aldrich (Paris, France). HPTLC plates $(20 \times 10 \mathrm{~cm}$, silica gel $60 \mathrm{~F} 254)$ were obtained from Merck (Darmstadt, Germany).

\subsection{Plant material}

Lentisk fruits were collected from Tabarka in North of Tunisia in September 2016 located at the following geographical coordinates obtained by GPS, $\left(36^{\circ} 5783,6^{\prime} \mathrm{N}\right.$ and $08^{\circ} 5736,2^{\prime} \mathrm{E}$, altitude: $20 \mathrm{~m}$ ). Fruits were air-dried in the dark at room temperature and were ground using MF 10 basic microfine grinder drive, IKA ${ }^{\circledR}$ (Rotar speed $6500 \mathrm{rpm}, \mathrm{MF}$ sieves of $2 \mathrm{~mm}$ of diameter).

\subsection{Lipid extraction}

Oils were isolated from Lentisk fruit by a Soxhlet extractor. Thirty gram of ground dried fruit were weighted inside a $30 \times 100 \mathrm{~mm}$ cellulose thimble (Macherey-Nagel, Düren, Germany) and loaded into the extraction chamber of a $125 \mathrm{~mL}$ Soxhlet extractor fitted with a condenser, which was extracted with $300 \mathrm{~mL}$ of solvent placed in a $500 \mathrm{~mL}$ distillation flask. Samples were extracted under reflux during $8 \mathrm{~h}$ with the selected solvents (hexane, EtOH, IPA, EtOAc, ethyl lactate, DMC, $p$-cymene, $\alpha$-pinene, limonene, MeTHF and CPME). All extractions were conducted in triplicate. Subsequently, solvents were evaporated under reduced pressure, using a rotary evaporator at $45^{\circ} \mathrm{C}$ except ethyl lactate, $p$-cymene, $\alpha$-pinene and limonene extracts. These solvents have a high boiling point $\left(154,177,155\right.$ and $176^{\circ} \mathrm{C}$, respectively) and their elimination are not possible by conventional evaporation under reduced pressure. So, to facilitate their evaporation, the principle of hetero-azeotropic distillation was applied. Thus, $50 \%(\mathrm{~V} / \mathrm{V})$ of water were added to $p$-cymene, $\alpha$-pinene and limonene extracts and $70 \%(\mathrm{~V} / \mathrm{V})$ of water were added to ethyl lactate extracts. Then, the mixtures were evaporated as described before. The dried residues were weighted and oils were aliquoted in vials and stored at $4{ }^{\circ} \mathrm{C}$ until analysis. Oil content was determined according to NF V 03-908 and was expressed as a percentage of the total weight of lipids obtained after extraction relative to the dry weight of Lentisk.

\subsection{Computational methods: COSMO-RS simulations}

COSMO-RS is a computational prediction model that combines a quantum chemical with thermodynamical statistics to determine and predict the chemical potential of molecules in a liquid without experimental data (Klamt, 2003). It is a powerful method used as a decision-making tool for solvent screening (Sicaire et al., 2015; Rapinel et al., 2016; Ravi et al., 2018).

COSMO-RS simulation consists on two-step. First, the microscopic step is where simulation is performed in a virtual 
conductor environment for the molecules. In this environment, molecule induced polarization charge density " $\sigma$ " on the surface ( $\sigma$-surface). Thus, the solute molecule is converted to its energetically optimal state, via the quantum calculation self-consistency algorithm triple zeta valence polarized basis set (TZVP), with respect to its geometry and electron density.

The second step used the statistical thermodynamic calculation. In this stage, the polarization charge density was used for the quantification of the interaction energy of the pair-wise interacting surface segments regarding electrostatics and hydrogen bonding. The polarization charge was converted into a surface composition function ( $\sigma$-profile) that gives information of the molecular polarity distribution. Thus, the $\sigma$-surface and the $\sigma$-profile allowed the characterization of a reference molecule (minimum energy, stable molecule) as a solute in a perfect conductor. The obtained $\sigma$-profile was then used to calculate the chemical potential of the surface segment $(\sigma$-potential) using COSMOthermX program (version C30 release 17.02). The affinity of a solvent and the interaction between solutes and solvent in mixed state can be predicted using the $\sigma$-profile and the $\sigma$-potential. The solutes were selected after exhaustive literature review. The relative solubility of target free fatty acids (palmitic, oleic and linoleic acids) and TAGs (palmitoyl-oleyl-linoleoylglycerol [POL], palmitoyl-dioleylglycerol [POO], dioleyl-linoleoyl-glcerol [OOL] and trioleylglycerol [OOO]) in different solvents (hexane, EtOH, IPA, DMC, EtOAc, CPME, MeTHF, limonene, $\alpha$-pinene, $p$-cymene and ethyl lactate) was calculated with COSMOtherm software (C30 1401, CosmothermX14, COSMOlogic $\mathrm{GmbH} \& \mathrm{Co} . \mathrm{KG}$ ) as follows:

$$
\log _{10}\left(x_{j}\right)=\log _{10}\left[\frac{\exp \left(\mu_{j}^{\text {pure }}-\mu_{j}^{\text {solvent }}-\Delta G_{j, \text { fusion })}\right.}{R T}\right],
$$

$\mu_{j}^{\text {pure }}:$ chemical potential of pure compound $j$ (joule/

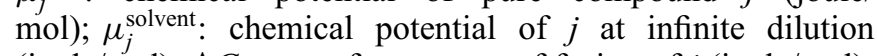
(joule/mol); $\Delta G_{j, \text { fusion) }}$ : free energy of fusion of $j$ (joule $/ \mathrm{mol}$ ); $x_{j}$ : solubility of $j(\mathrm{~g} / \mathrm{g}$ solvent).

Relative solubility is calculated in infinite dilution. The logarithm of the best solubility is set to 0 and all other solvents are ranked relatively to the best or reference solvent.

\subsection{Qualitative and quantitative analyses of lipids}

\subsubsection{High performance thin layer chromatography} (HPTLC)

Polar and neutral lipids of Lentisk oil were detected by charring and quantified with a CAMAG 3 TLC densitometer (CAMAG, Muttenz, Switzerland) through two different HPTLC developments. Classes of lipids were identified in comparison with known polar and neutral lipid standards. Plates were conditioned with a mixture of chloroform/ methanol $(2: 1, \mathrm{v} / \mathrm{v})$ and activated at $110^{\circ} \mathrm{C}$ for $60 \mathrm{~min}$ on the TLC Plate Heater (CAMAG, Muttenz, Switzerland). The oil samples were spotted onto $20 \times 10 \mathrm{~cm}$ HPTLC plates (Silica gel 60 F254; Merck KGaA, Darmstadt, Germany) by means of an ATS 5 automatic TLC sampler (CAMAG, Switzerland). Plates were developed in an ADC2 automatic developing chamber (CAMAG). The separation of neutral lipids was performed with hexane/diethyl ether/glacial acetic acid in a ratio of $65: 35: 2(\mathrm{v} / \mathrm{v} / \mathrm{v})$ to a height of $85 \mathrm{~mm}$. For polar lipids, the eluent system was a mixture of methylacetate/ isopropanol/chloroform $/ \mathrm{methanol} / \mathrm{KCl}(0.25 \%$ solution $)$ in a ratio of $25: 25: 25: 10: 9(\mathrm{v} / \mathrm{v} / \mathrm{v} / \mathrm{v} / \mathrm{v})$ running to a height of $85 \mathrm{~mm}$. The dried plate was dipped for $6 \mathrm{~s}$ in a reagent $(10 \mathrm{mg}$ of primuline, $160 \mathrm{~mL}$ of acetone and $40 \mathrm{~mL}$ of water) and then scanned using a TLC Scanner 3 with a WinCATs software (CAMAG). Results are expressed as percentage of lipid classes in total Lentisk fruit oil (LFO).

\subsubsection{Fatty acid methyl ester (FAME) preparation and gas chromatography analysis}

Before the injection of samples in GC-FID, fatty acid methyl esters (FAMEs) were prepared from the extracted oil by means of acid-catalyzed trans-methylation as reported by Morrison and Smith (1964). One millimeter of methanolic sulfuric acid $(5 \% \mathrm{v} / \mathrm{v})$ was mixed with a determined quantity of extracted oil and $400 \mu \mathrm{L}$ of Triheptadecanoin (C17:0 TAG) used as internal standard. The mixture was heated at $85^{\circ} \mathrm{C}$ for $90 \mathrm{~min}$. After cooling, $1 \mathrm{~mL}$ of hexane and $1.5 \mathrm{~mL}$ of sodium chloride $(0.9 \%)$ solution were added to the mixture and shaken vigorously during $30 \mathrm{~s}$. Two microliter of the top organic layer was injected in split mode (split ratio: 1:20) at $250{ }^{\circ} \mathrm{C}$ onto a $7820 \mathrm{~A}$ gas chromatography system (Agilent technologies, USA) equipped with flame ionization detector and a BD-EN14103 capillary column $(30 \mathrm{~m} \times 320 \mu \mathrm{m} \times 0.25$ $\mu \mathrm{m})$. Helium was used as a carrier gas at $33 \mathrm{~cm} \mathrm{~s}^{-1}$. The oven temperature was programmed as follows: firstly, temperature was maintained at $50^{\circ} \mathrm{C}$ for $1 \mathrm{~min}$, then increased to $220^{\circ} \mathrm{C}$ at a rate of $20^{\circ} \mathrm{C} / \mathrm{min}$ and reached $280^{\circ} \mathrm{C}$ at a rate of $2^{\circ} \mathrm{C} / \mathrm{min}$. Finally, it was maintained isothermally for $10 \mathrm{~min}$ at $230^{\circ} \mathrm{C}$. FAMEs were identified by comparing their retention time with those of purified standards (Sigma Co., St. Louis, MO, USA) using Agilent EZChrom Elite software.

\subsection{Chemometric data treatment}

GC-FID and HPTLC data were analyzed using SIMCA software version 14.1.0.2047 (Umetrix, Umeå, Sweden), a multivariate statistical analytical software, for unsupervised Principal Component Analysis (PCA) and Hierarchical Cluster Analysis (HCA). For HCA analysis, similarities between the different samples were estimated by means of the Ward linkage distance algorithm. Similar samples were characterized by small distances and the opposite for dissimilar samples. The data matrix was subjected to $\log$ transformation before the multivariate analysis.

\subsection{Measurement of the anti-inflammatory activity by nitrite quantification}

\subsubsection{Cell culture}

RAW 264.7 murine macrophage cells were supplied from the American Type Culture Collection (ATCC, Manassas, USA). The cell line was cultured in RPMI 1640 medium supplemented with $10 \%(\mathrm{v} / \mathrm{v})$ fetal bovine serum, $100 \mathrm{U} / \mathrm{mL}$ of penicillin, $100 \mu \mathrm{g} / \mathrm{mL}$ of streptomycin and were maintained at $37^{\circ} \mathrm{C}$ in humidified atmosphere of $5 \%$ carbon dioxide. 
Table 1. Conductor-like screening model for real solvent (COSMO-RS) assisted solvent screening: relative solubility (log10 [Xsolub]) of major compounds of Pistacia lentiscus fruit oil in several solvents. The logarithm, $\log 10$ (Xsolub), of the best solubility is fixed to zero and the rest of solvents are given relatively to the best one.

\begin{tabular}{|cccccccc|}
\hline & \multicolumn{3}{c}{ FFAs } & \multicolumn{5}{c|}{ TAGs } \\
\hline Solvents & FFA1 & FFA2 & FFA3 & TAG1 & TAG2 & TAG3 & TAG4 \\
\hline Hexane & -1.27 & -1.34 & -1.31 & 0 & 0 & -0.17 & -0.001 \\
EtOH & -0.001 & -0.05 & 0 & -2.52 & -2.53 & -2.19 & -2.55 \\
IPA & 0 & 0 & 0 & -1.49 & -1.51 & -1.24 & -1.52 \\
EtOAc & 0 & 0 & 0 & 0 & 0 & 0 & 0 \\
Ethyl lactate & -0.22 & -0.27 & -0.06 & -3.23 & -3.24 & -3.99 & -3.15 \\
DMC & -0.56 & -0.51 & -0.22 & -1.51 & -1.46 & -1.21 & -1.41 \\
$p$-cymene & -1.03 & -1.03 & -0.9 & 0 & 0 & 0 & 0 \\
$\alpha$-pinene & -1.08 & -1.11 & -1.03 & 0 & 0 & 0 & 0 \\
Limonene & -0.96 & -0.98 & -0.88 & 0 & 0 & 0 & 0 \\
MeTHF & 0 & 0 & 0 & 0 & 0 & 0 & 0 \\
CPME & 0 & 0 & 0 & 0 & 0 & 0 & 0 \\
\hline
\end{tabular}

Solubility index Reference or equivalent Better than reference Lesser than reference

FFAs: FFA1 (C16:0); FFA2 (C18:1); FFA3 (C18:2). TAGs: TAG1: OOO (C18:1 × 3); TAG2: OOL (C18:1 × 2+ C18:2); TAG3: POO $(\mathrm{C} 16: 0+\mathrm{C} 18: 1 \times 2) ;$ TAG4: POL $(\mathrm{C} 16: 0+\mathrm{C} 18: 1+\mathrm{C} 18: 2)$.

\subsubsection{Measurement of nitrite production}

RAW 264.7 cells were seeded in $24-$ well plates at a density of $2 \times 105$ cells per well and were allowed to attach for $24 \mathrm{~h}$ at $37^{\circ} \mathrm{C}$.

Then, after $60 \mathrm{~min}$, treated cells with increasing concentrations of LFO extracts, dissolved in the DMSO, were stimulated with $100 \mu \mathrm{g} / \mathrm{mL}$ lipopolysaccharide (LPS). After $24 \mathrm{~h}$, the quantity of nitrite accumulated in the culture supernatant was determined based on the Griess reaction (Aboura et al., 2017). The absorbance at $540 \mathrm{~nm}$ was then measured and nitric oxide (NO) levels, produced by murine macrophage-like RAW264.7 cells, were determined by comparison with a $\mathrm{NaNO}_{2}$ standard curve.

\section{Results and discussion}

\subsection{In silico screening}

Simulations showed that all bio-based solvents have a good potency to replace hexane for the extraction of FFAs (Tab. 1). In fact, green solvents have a high solubilization power than hexane, as $\log 10$ (Xsolub) of green solvents are higher than $\log 10$ (Xsolub) of hexane. Regarding the $\log 10$ (Xsolub) of TAGs, EtOAc, $p$-cymene, $\alpha$-pinene, limonene, MeTHF and CPME are equal to 0 , endorsed as good solvent candidates for TAGs extraction. Nevertheless, EtOH, IPA, Ethyl lactate and DMC are theoretically not suitable for TAGs extraction compared to hexane as solubility values are below zero.

According to the in silico screening, MeTHF, EtOAc and CPME seem to be the most suitable alternative solvents for the extraction of LFO major metabolites.

\subsection{Experimental solvent screening}

To validate theoretical COSMO-RS predictions, the performance of alternative solvents to substitute hexane for the extraction of LFO was tested experimentally.

Concerning lipid classes, HPTLC analyses of LFO obtained by green solvents and hexane as reference are illustrated in Table 2. Results showed that the major lipids extracted with both alternative and conventional solvents were TAGs (72.1699.99\%). Figure 1a exhibited the unsupervised PCA biplot representing PC2 versus $\mathrm{PC} 1$ with 54.5 and $23.6 \%$ respectively, explaining a total variance of $78.1 \%$. MeTHF, CPME, EtOAc, DMC, $p$-cymene and hexane extracts are located near TAGs. IPA and EtOH were closer to phospholipids (Lyso, PI, PC and PE) and were closely separated from the other solvents along PC1 and PC2. This indicates that alcohols have a good solvation power of polar lipids. In fact, the presence of these phospholipids in alcohol extracts can be explained by the high polarity of these solvents compared to hexane (Breil et al., 2016). On the other hand, ethyl lactate, limonene and $\alpha$-pinene were separated from alcohol solvents along PC1 and from other solvents along PC2 and were closer to MAG and DAG. In fact, these solvents have a different lipid class profile than the others, characterized by a high content of DAG $(19.73,12.68$ and $8.29 \%$ for ethyl lactate, limonene and $\alpha$-pinene, respectively). Furthermore, a higher MAG content was detected in ethyl lactate extract $(8.10 \%)$ than hexane $(2.24 \%)$. These solvents have a high boiling point which could affect their efficiency and induce lipid degradation (Cascant et al., 2017).

Extracted oils were then analyzed by GC-FID after transmethylation of fatty acids. LFO were mainly composed 
E. Chaabani et al.: OCL 2019, 26, 25

Table 2. Lipid classes of Pistacia lentiscus fruit oil obtained by various solvents.

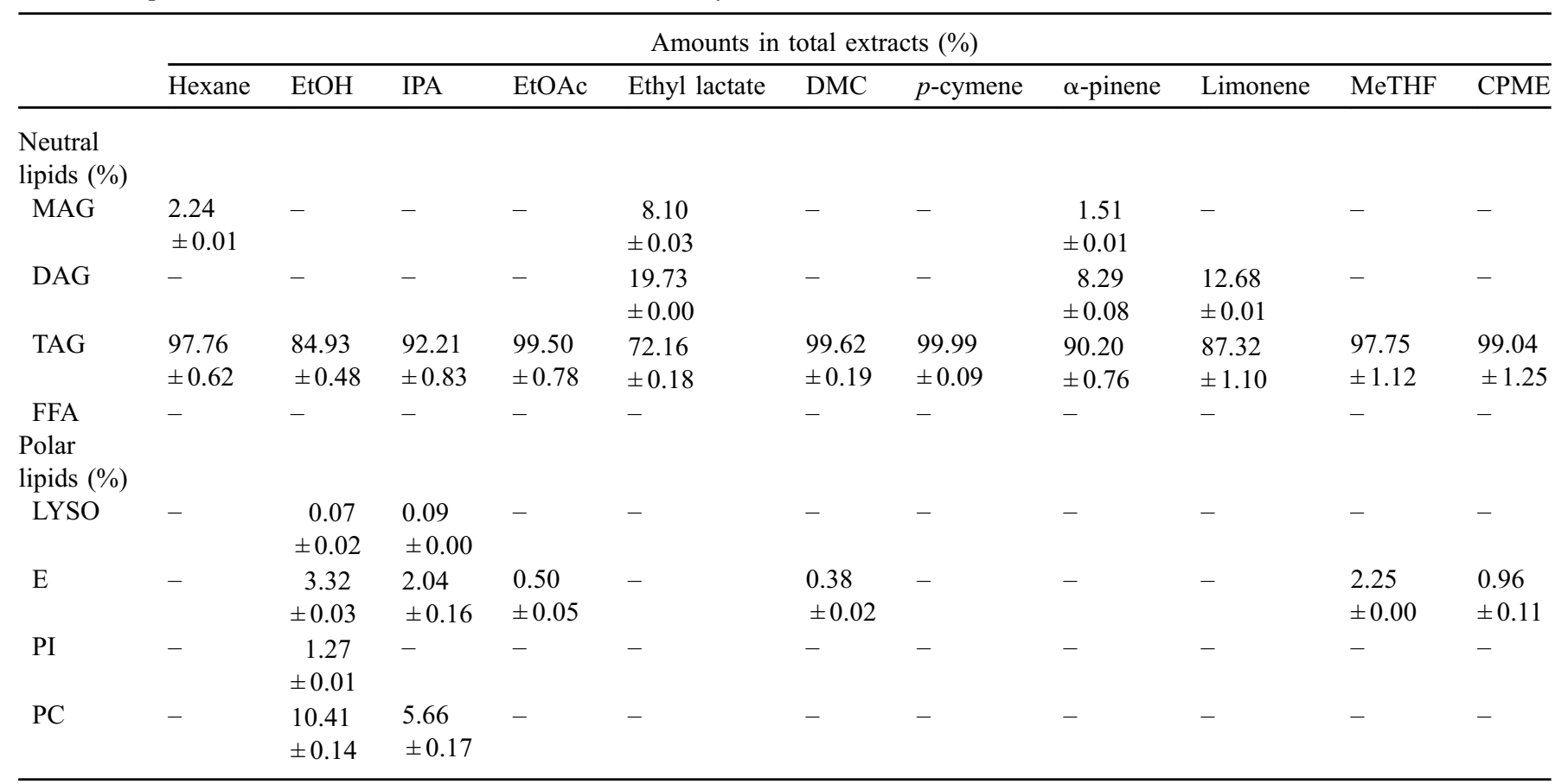

Data are expressed as mean \pm SE of triplicate samples. MAG: monoacylglycerol. DAG: diacylglycerol. TAG: triacylglycerol. FFA: free fatty acid. LYSO: lysophosphatidilcholine. PE: phosphatdylethanolamine. PI: phosphatidylinositol. PC: phosphatydylcholine.

by oleic acid (C18:1n9) (41.8-48.37\%), linoleic acid (C18:2n6) (22.21-26.1\%) and palmitic acid (C16) (22.11$23.96 \%$ ) (Tab. 3). These results are in agreement with previous studies (Dhifi et al., 2013; Mezni et al., 2014).

To assess the influence of the solvent extraction on overall metabolites, GC-FID data of LFO obtained using the different studied solvents were applied to PCA. PCA model (Fig. 1b) indicated that the two first principal components explain $55.7 \%$ of the total variance with 34.6 and $21.1 \%$ for PC1 and PC2, respectively. PCA data exhibited that $p$-cymene was closely separated from hexane, MeTHF, IPA, EtOH, ethyl lactate, EtOAc and DMC along the PC1 and from CPME and $\alpha$-pinene along PC2. $\alpha$-Pinene was located in the opposite side of hexane indicating a different metabolite profile. However, it can be noticed that hexane, MeTHF, CPME, ethyl lactate, limonene, EtOH, IPA, EtOAc and DMC were located in the middle of all FFAs.

Obviously, the PCA provides a multivariate overview of the tendency of clustering/separation of samples due to their metabolite profiles, but cannot show the underlying degree of similarities between clusters. So, Hierarchical Cluster Analysis (HCA) was performed on GC-FID data using Ward linkage distance method. The resulting HCA dendrogram (Fig. 2) showed that the dissolving power of tested solvents could be classified into four different clusters. $\alpha$-pinene and $p$-cymene formed the two first clusters. The third cluster was composed by CPME, limonene, ethyl lactate and $\mathrm{EtOH}$ and the fourth contained hexane, MeTHF, IPA, EtOAc and DMC. From these results, it can be concluded that MeTHF, IPA, EtOAc and DMC extracts have similar fatty acid profiles compared to hexane extracts. In contrast, $p$-cymene and $\alpha$-pinene showed the highest dissimilarity to the other two clusters.

Yields were around 6.14 and $8.61 \%$ (Fig. 3). No significant difference between hexane, EtOH, EtOAc, ethyl lactate, DMC, limonene and MeTHF was observed. CPME, $p$-cymene and $\alpha$-pinene resulted in the lowest lipid yields $(6.65,6.14$ and $5.67 \%$ respectively). In contrast, IPA yields were relatively higher than hexane but this may be related to the higher extraction of phospholipids as shown in HPTLC analyses.

\subsection{In silico screening versus experimental screening}

The theoretical COSMO-RS results were compared to the experimental data. The COSMO-RS prediction indicated that MeTHF, CPME, and EtOAc were theoretically the most suitable solvents to replace hexane. However, taken into account global yield, lipid composition and statistical analysis, the experiments showed that among these 3 predicted alternative solvents, only MeTHF and EtOAc were the suitable solvents for hexane. Furthermore, DMC appeared to be a good candidate to replace hexane experimentally, which was not in accordance with theoretical screening. Experimentally, CPME gives the lowest lipid yields and was not a good alternative to replace hexane.

The selection of an alternative solvent has to consider parameters other than solubility, as $\log P$, boiling point, energy required for the evaporation of the solvent and toxicity index (Verpoorte et al., 2008; Ernst et al., 2014). Indeed, based on solubility matching, economic and energy parameters MeTHF was found to be the best alternative bio-based solvent 

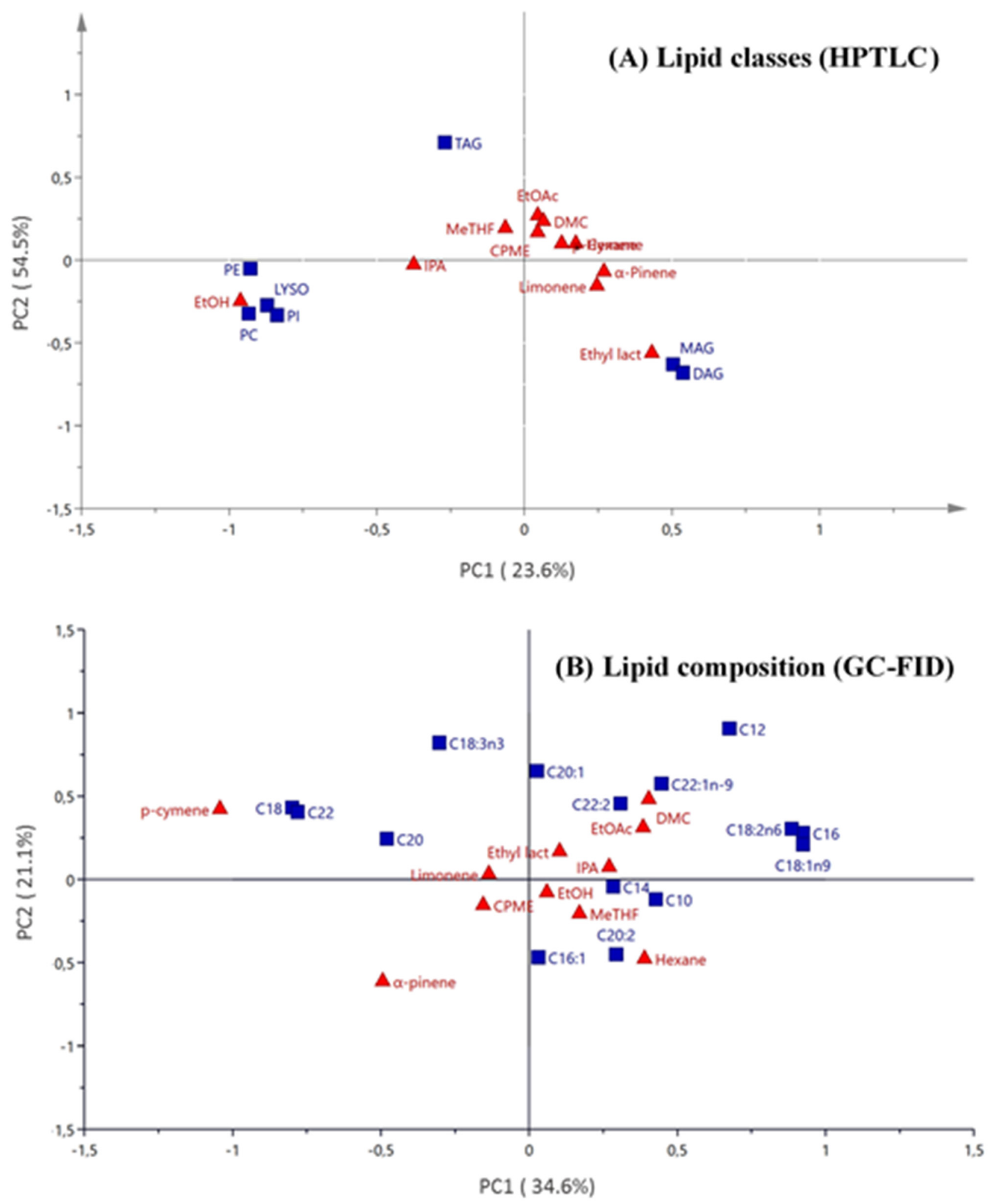

Fig. 1. Principal component analysis biplot (score and loading values) represented by PC1 versus PC2 for (A) lipid classes obtained by HPTLC and (B) lipid composition obtained by GC-FID, and tested solvents.

to replace hexane for the extraction of LFO. This bio-based solvent derived from renewable source has a lower toxicity (Clarke et al., 2018) and its use in pharmaceutical chemical processes has been approved by Antonucci et al. (2011). Also, this solvent required practically the same energy to evaporate $1 \mathrm{~kg}$ of solvent as hexane, low boiling point and $\log P>0$. 


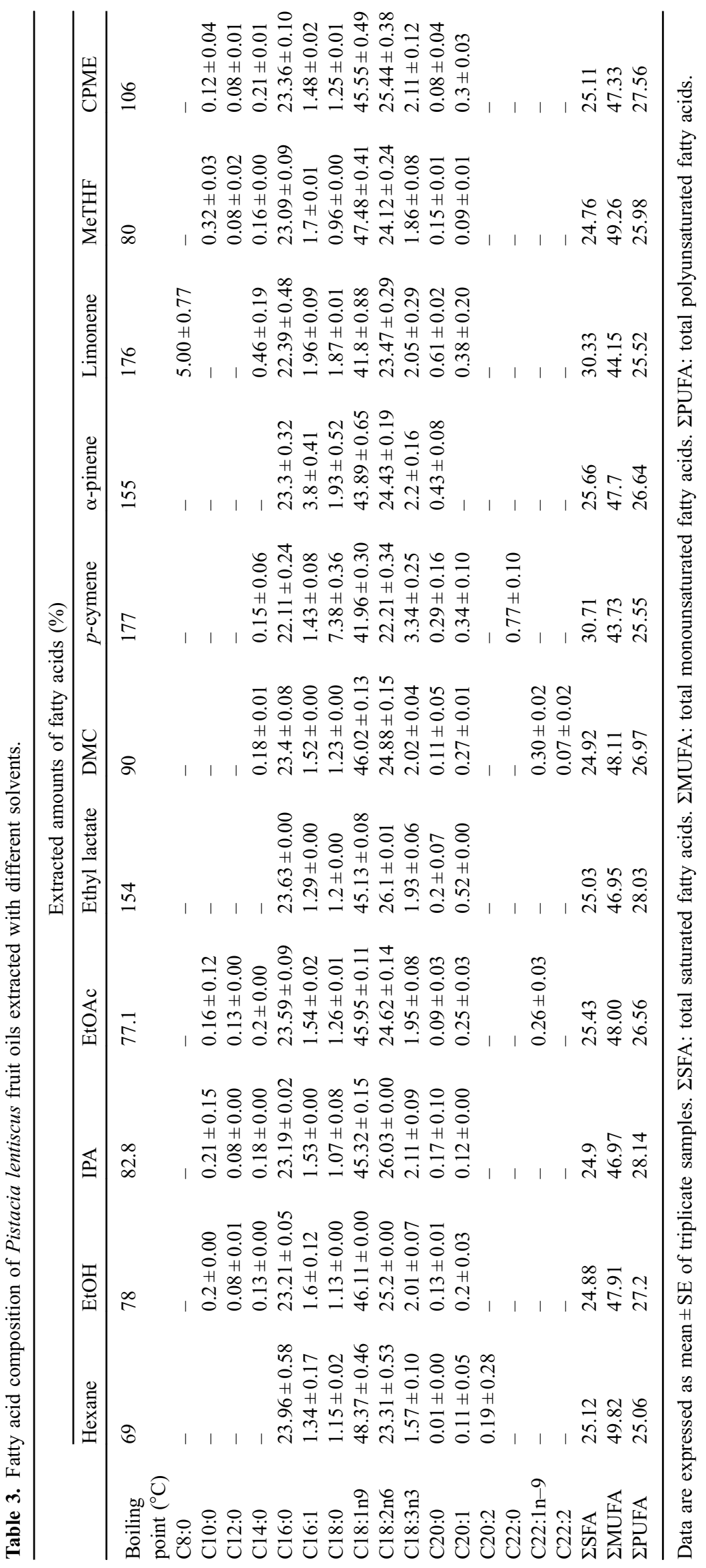




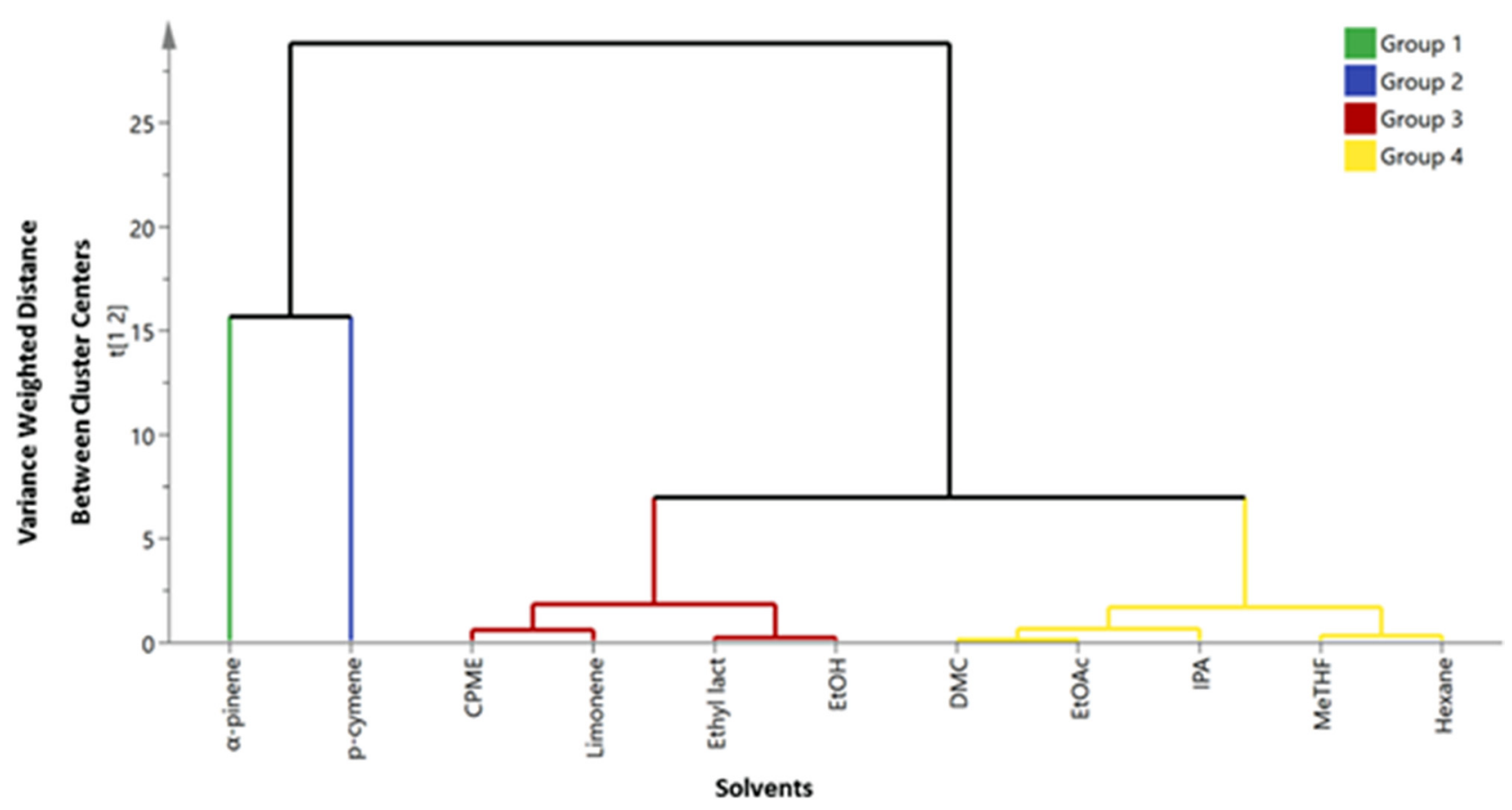

Fig. 2. Cluster dendrographic classification of tested solvents based on their GC-FID results established using Ward's linkage distance.

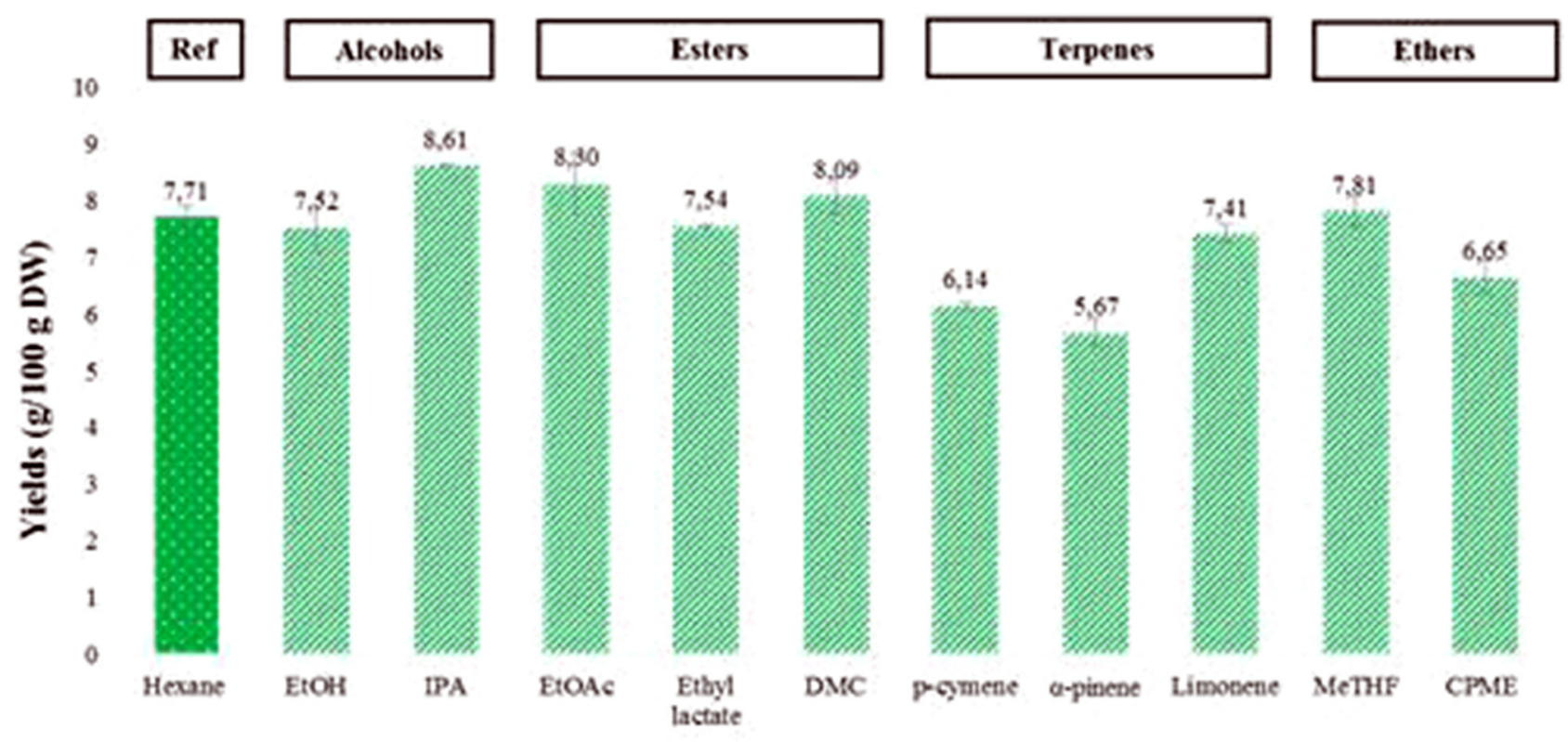

Solvents

Fig. 3. Comparison of lipid extraction yields obtained by bio-based solvents compared to the reference.

Nevertheless, DMC and EtOAc required more energy to evaporate $1 \mathrm{~kg}$ of solvent $(0.194 \mathrm{KW} / \mathrm{h}, 0.217 \mathrm{~kW} / \mathrm{h})$ compared to hexane $(0.121 \mathrm{KW} / \mathrm{h})$.

\subsection{In vitro anti-inflammatory activity}

During the inflammatory process, macrophage produces mediators, such as nitric oxide (NO) by inducible NO synthase
(iNOS). Thus, NO is recognized as a mediator and regulator in acute inflammatory responses. An excessive production of $\mathrm{NO}$ induces a variety of inflammation and carcinogenesis aspects. In the present study, the anti-inflammatory activity of MeTHF lipid extract, which was found to be the best alternative solvents to substitute hexane for the extraction of LFO, was investigated using LPS-activated RAW 264.7 macrophages. Firstly, the cytotoxicity of MeTHF lipid extract was evaluated. 


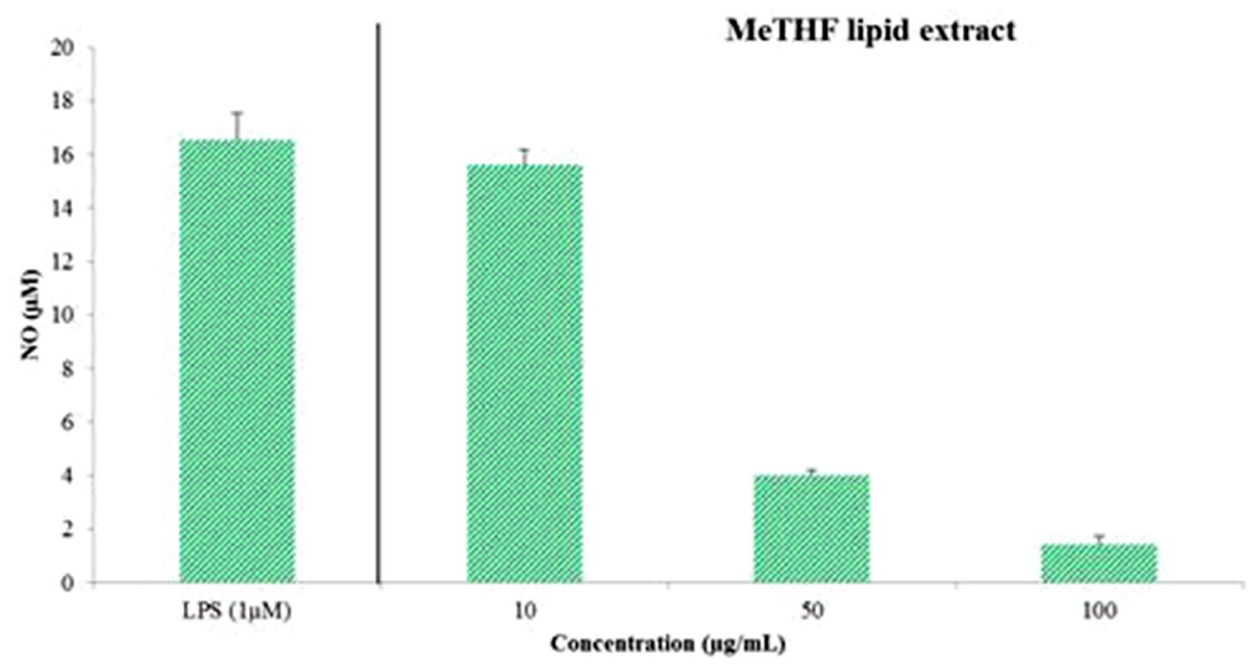

Fig. 4. Effect of Pistacia lentiscus oil obtained with MeTHF on NO overproduction in LPS-stimulated RAW264.7 macrophages. Each value represents the mean $\pm \mathrm{SD}$ of triplicate.

RAW 267.4 cells were treated with increasing concentrations of MeTHF lipid extract $(10-200 \mu \mathrm{g} / \mathrm{mL})$. MeTHF lipid extracts did not reveal any significant cytotoxicity against RAW 267.4 macrophage cells until $100 \mu \mathrm{g} / \mathrm{mL}$ (data not shown). In fact, our experiments were carried out at the nontoxic concentrations of 10,50 and $100 \mu \mathrm{g} / \mathrm{mL}$. Then, the effect of MeTHF lipid extracts on nitrite secretion was investigated. As shown in Figure 4, MeTHF extract decreased NO release in a dose-dependent manner. In fact, NO levels in RAW 267.4 cells decreased from $15.6 \mu \mathrm{M}(10 \mu \mathrm{g} / \mathrm{mL})$ to $1.4 \mu \mathrm{M}(100 \mu \mathrm{g} / \mathrm{mL})$, while NO production in LPS-activated cells, not treated with LFO, was higher $(16.5 \mu \mathrm{M})$ than treated cells. These results suggest the important anti-inflammatory activity of MeTHF lipid extract. In fact, MeTHF lipid extract was mainly composed by oleic and linoleic acid (47.48 and $24.12 \%$, respectively). These two unsaturated fatty acids have anti-inflammatory properties playing a major role in the recruitment of inflammatory cells at the inflammation site (Ben Khedir et al., 2017).

\section{Conclusion}

The potential of alternative green solvents to replace hexane for lipid extraction from Pistacia lentiscus L. berries was sought and the anti-inflammatory activity of LFO obtained with the most suitable alternative solvent was evaluated herein. Some discrepancies between theoretical and experimental screening were noticed. For COSMO-RS simulation MeTHF, EtOAC and CPME were the best suitable solvents for hexane. Nevertheless, experiments highlighted that MeTHF was the best alternative solvent compared to hexane qualitatively and quantitatively. Our data approved the potential of alternative solvents especially MeTHF to substitute the toxic hexane, conventionally used for lipid extraction.

Results also highlighted the great nutritional value of LFO regarding the high amount of oleic and linoleic acid which may protect against some metabolic disorders, cardiovascular and cancer diseases (Trabelsi et al., 2012) and demonstrated its strong anti-inflammatory activity in RAW 264.7 macrophages.
These results would encourage the use of LFO in food industry as edible oil and also its valorization in pharmaceutical and cosmetics manufactory.

Conflicts of interest. The authors declare that they have no conflicts of interest in relation to this article.

\section{References}

Abouraa I, Nani A, Belarbi M, et al. 2017. Protective effects of polyphenol-rich infusions from carob (Ceratonia siliqua) leaves and cladodes of Opuntia ficus-indica against inflammation associated with diet-induced obesity and DSS-induced colitis in Swiss mice. Biomed Pharmacother 96: 1022-1035.

Antonucci V, Coleman J, Ferry JB, et al. 2011. Toxicological assessment of 2-methyltetrahydrofuran and cyclopentyl methyl ether in support of their use in pharmaceutical chemical process development. Org Process Res Dev 15: 939-941.

Ben Khedir S, Bardaa S, Chabchoub N, Moalla D, Sahnoun Z, Rebai T. 2017. The healing effect of Pistacia lentiscus fruit oil on laser burn. Pharm Biol 55: 1407-1414.

Breil C, Meullemiestre A, Vian M, Chemat F. 2016. Bio-based solvents for green extraction of lipids from oleaginous yeast biomass for sustainable aviation biofuel. Molecules 21: 196. doi: 10.3390/molecules21020196.

Cascant MM, Breil C, Miguel de la Guardia SG, Fabiano-Tixier AS, Chemat F. 2017. A green analytical chemistry approach for lipid extraction: Computation methods in the selection of green solvents as alternative to hexane. Anal Bioanal Chem doi: 10.1007/s00216-017-0323-9.

Clarke CJ, Tu WC, Levers O, Bröhl A, Hallett JP. 2018. Green and sustainable solvents in chemical processes. Chem Rev 118: 747800.

Dhifi W, Jelali N, Chaabani E, et al. 2013. Chemical composition of Lentisk (Pistacia lentiscus L.) seed oil. Afr J Agric Res 8: 1395 1400 .

Ernst M, Silva DB, Silva RR, Vêncio RZN, Lopes NP. 2014. Mass spectrometry in plant metabolomics strategies: From analytical platforms to data acquisition and processing. Nat Prod Rep 31: 784-806. 
Kerton FM, Marriott R. 2013. Alternative solvents for green chemistry. Croydon: Royal Society of Chemistry.

Klamt A. 2003. Prediction of the mutual solubilities of hydrocarbons and water with COSMO-RS. Fluid Phase Equilib 206: 223-235.

Mezni F, Labidi A, Msallem M, Boussaid M, Khouja ML, Khaldi A. 2014. Influence of harvest date on fatty acid composition and antioxidant activity of Pistacia lentiscus L. edible oils. J Mater Environ Sci 5: 1703-1708.

Mezni F, Shili S, Ben Ali N, Larbi Khouja M, Khaldi A, Maaroufi A. 2016. Evaluation of Pistacia lentiscus seed oil and phenolic compounds for in vitro antiproliferative effects against BHK21 cells. Pharm Biol 54: 747-751.

Morrison WR, Smith LM. 1964. Preparation of fatty acid methyl esters and dimethylacetals from lipids with boron fluoride methanol. J Lipid Res 5: 600-608.

Naouar MS, Zouiten Mekki L, Charfi L, Boubaker J, Filali A. 2016. Preventive and curative effect of Pistacia lentiscus oil in experimental colitis. Biomed Pharmacother 83: 577-583.
Rapinel V, Rombaut N, Rakotomanomana N, Vallageas A, Cravotto G, Chemat F. 2016. An original approach for lipophilic natural products extraction: Use of liquefied n-butane as alternative solvent to n-hexane. LWT-Food Sci Technol 85: 524-533.

Ravi HK, Breil C, Vian MA, Chemat F, Venskutonis PR. 2018. Biorefining of Bilberry (Vaccinium myrtillus L.) Pomace using microwave hydrodiffusion and gravity, ultrasound-assisted, and bead-milling extraction. ACS Sustain Chem Eng 6: 4185-4193.

Sicaire AG, Abert Vian M, Fine F, Carré P, Tostain S, Chemat F. 2015. Experimental approach versus COSMO-RS assisted solvent screening for predicting the solubility of rapeseed oil. OCL 22: D404.

Trabelsi H, Cherif OA, Sakouhi F, et al. 2012. Total lipid content, fatty acids and 4-desmethylsterols accumulation in developing fruit of Pistacia lentiscus L. growing wild in Tunisia. Food Chem 131: 2-6.

Verpoorte R, Choi YH, Mustafa NR, Kim HK. 2008. Metabolomics: Back to basics. Phytochem Rev 7: 525-537.

Cite this article as: Chaabani E, Abert Vian M, Dakhlaoui S, Bourgou S, Chemat F, Ksouri R. 2019. Pistacia lentiscus L. edible oil: green extraction with bio-based solvents, metabolite profiling and in vitro anti-inflammatory activity. OCL 26: 25. 